\title{
Improvement of the Nutritional Value of a Cereal Fermented Milk: 2-Dried Kishk Like
}

\section{Nassar KS ${ }^{1 *}$, Shamsia $\mathrm{SM}^{1}$ and Attia $\mathrm{IA}^{2}$}

${ }^{1}$ Department of Food and Dairy Science and Technology, Damanhour University, Egypt ${ }^{2}$ Department of Dairy Science and Technology, Alexandria University, Egypt

\begin{abstract}
The present study has been conducted to produce fermented milk fortified with different cereals like whole wheat, barley and freek (green wheat) burghul have been selected for their known nutrition benefits. The fermentation was occurred by using three types of cultures, yogurt starter, yogurt starter + Bio-yogurt or yogurt starter + Lactobacillus plantarum. All samples were stored at room temperature $\left(25 \pm 2^{\circ} \mathrm{C}\right)$ for three months and have been subjected to consumer sensory testing; dried kishk-like products were highly accepted by the tasting panel, furthermore fermented dairy products containing Freek gained the highest score of judging followed by wheat. Proximate composition, Colour, Organic acids and microbiologically analysis have been monitored in the fresh soft product and during storage. Nevertheless, the combined levels of organic acids, low pH, salt additive and low moisture content in the kishk samples were sufficient to ensure the microbial safety of the product. Thus, long shelf-life of all samples without changing in their chemical during the storage period has been noticed.
\end{abstract}

Keywords: Burghul; Probiotic bacteria; Dried kishk; Organic acids; Colour; Skim milk

\section{Introduction}

Fermentation is one of the oldest and most economical methods of producing and preserving food. Also, leads to a general improvement in the shelf life, texture, taste and aroma of the final product. Fermented foods are produced world-wide using various manufacturing techniques, raw materials and microorganisms [1]. Kishk is an extremely popular fermented food in many parts of the Middle East. In Egypt, Kishk is one of the traditional food products in Upper Egypt $[2,3]$. There are some other products similar to kishk such as tarhana (mixing yogurt, wheat flour, baker's yeast and variety of vegetables and spices in turkey), kushuk (milk- sour dough mixture with turnips in Iraq), atole (fermented cereal-milk porridge in Scotland and Greece) and tahonya/talkuna (fermented cereal mixture with vegetables in Finland and Hungary) [4]. Kishk is a natural, healthy, respect the environment and have great taste and cultural values that are increasingly attractive to the Egyptian consumers. Kishk made by mixing wheat with fermented milk (Laban zeer) and sun-drying the mixture to $8 \%$ to $12 \%$ moisture content $[2,5]$ or made from different cereal products and fermented milk base by traditional methods of manufacture [6,7]. Kishk is usually reconstituted with water and served as a hot gruel, but with the incorporation of vegetables, spices, garlic or herbs, can form the base of savory and sweet dishes [8]. The aim of the present study was to focus some light on the chemical, biochemical, physical and sensory aspects of dried fermented milk made from various types of cereal and starter cultures.

\section{Materials and Methods}

In the previous paper, the preparation kishk-like from reconstituted skim milk and burghul from whole wheat, whole barley and fereek was described in Nassar et al. [9].

\section{Dried Kishk-like manufacturing}

Each type of burghul was mixed with reconstituted skim milk in a ratio of $1: 4(\mathrm{w} / \mathrm{w})$ in addition to, $2 \%$ sodium chloride and then mixed thoroughly each $26 \%$. Mixture was heated to $95^{\circ} \mathrm{C}$ for 10 seconds, and then rapidly cooled to $45^{\circ} \mathrm{C}$, addition $3 \%$ of each Starter. The resultant paste was filled in polystyrene cups and covered then incubated at $\left(43^{\circ} \mathrm{C}\right.$ for $\mathrm{W} 1, \mathrm{~B} 1$ and $\left.\mathrm{F} 1\right)$ and $\left(37^{\circ} \mathrm{C}\right.$ for $\mathrm{W} 2, \mathrm{~W} 3, \mathrm{~B} 2, \mathrm{~B} 3, \mathrm{~F} 2$ and $\mathrm{F} 3$ ) to 6 hours (Table 1). After that, the fermented paste was formed into nuggets $(3-5 \mathrm{~cm})$, placed into stainless steel trays and dried in air oven at $50^{\circ} \mathrm{C}$ for $15-18 \mathrm{~h}$. the dried nuggets were milled by using a hammer mill. After that, the dried kishk were stored in airtight glass containers and kept at room temperature until tested (Figure 1).

\section{Chemical analysis}

In the previous paper, the same methods were adopted for cereal and dairy base analysis as described in Nassar et al. [9].

\section{Dried Kishk-like analysis}

Proximate composition: Total solids, protein, ash and fiber content were determined according to procedures described by AOAC [10]. The fat content, salt percentage, acidity and $\mathrm{pH}$ value were determined according to Ling [11]. Finally, carbohydrate was calculated as follows [12]:

Carbohydrate $=$ total solids $-($ protein + fat + ash $)$

Colour of kishk-like samples: Colour of dried kishk samples was evaluated by Lovibond Schofield Tintometer (the Tintometer Ltd. Salisbury, England). Colours of samples were assessed. Reading obtained was further converted into C.I.E. (Commission International de L'E Clairage) units using the visual density graphs and direction booklet supplied with apparatus as described in AOCS [13].

*Corresponding author: Khaled S Nassar, Department of Food and Dairy Science and Technology, Damanhour University, Egypt, Tel: +20-453368069; E-mail: Khalid.nassar@agr.dmu.edu.eg

Received November 04, 2016; Accepted November 22, 2016; Published November 29, 2016

Citation: Nassar KS, Shamsia SM, Attia IA (2016) Improvement of the Nutritional Value of a Cereal Fermented Milk: 2-Dried Kishk Like. J Food Process Technol 7 638. doi: 10.4172/2157-7110.1000638

Copyright: @ 2016 Nassar KS, et al. This is an open-access article distributed under the terms of the Creative Commons Attribution License, which permits unrestricted use, distribution, and reproduction in any medium, provided the original author and source are credited. 
Citation: Nassar KS, Shamsia SM, Attia IA (2016) Improvement of the Nutritional Value of a Cereal Fermented Milk: 2-Dried Kishk Like. J Food Process Technol 7: 638. doi: 10.4172/2157-7110.1000638

Page 2 of 7

\begin{tabular}{|c|c|c|c|c|c|}
\hline \multirow{2}{*}{ Treatments } & \multicolumn{3}{|c|}{ Cereals } & \multirow{2}{*}{\begin{tabular}{|c|} 
Dairy base \\
Re-constituted Skim milk $(15 \%)$
\end{tabular}} & \multirow[t]{2}{*}{ Salt $2 \%$} \\
\hline & Whole Wheat Burghul (W) & Whole Barley Burghul (B) & Freek Burghul (F) & & \\
\hline \multirow{3}{*}{1} & $\sqrt{ }$ & & & $\sqrt{ }$ & $\sqrt{ }$ \\
\hline & & $\sqrt{ }$ & & $\sqrt{ }$ & $\sqrt{ }$ \\
\hline & & & $\sqrt{ }$ & $\sqrt{ }$ & $\sqrt{ }$ \\
\hline \multirow{3}{*}{2} & $\sqrt{ }$ & & & $\sqrt{ }$ & $\sqrt{ }$ \\
\hline & & $\sqrt{ }$ & & $\sqrt{ }$ & $\sqrt{ }$ \\
\hline & & & $\sqrt{ }$ & $\sqrt{ }$ & $\sqrt{ }$ \\
\hline \multirow{3}{*}{3} & $\sqrt{ }$ & & & $\sqrt{ }$ & $\sqrt{ }$ \\
\hline & & $\sqrt{ }$ & & $\sqrt{ }$ & $\sqrt{ }$ \\
\hline & & & $\sqrt{ }$ & $\sqrt{ }$ & $\sqrt{ }$ \\
\hline \multicolumn{6}{|c|}{$\begin{array}{l}\text { 1: }(3 \% \text { Yoghurt starter }) \\
2:(2 \% \text { Yoghurt starter }+1 \% \text { Bio-yoghurt starter }) \\
3:(2 \% \text { Yoghurt starter }+1 \% \text { Lactobacillus plantarun }\end{array}$} \\
\hline
\end{tabular}

Table 1: Experimental treatments

Reconstituted skim milk (15 W/W)

Wheat Burghul

Heat treatment at $95^{\circ} \mathrm{C} / 10 \mathrm{Sec}$ cooled to $45^{\circ} \mathrm{C}$
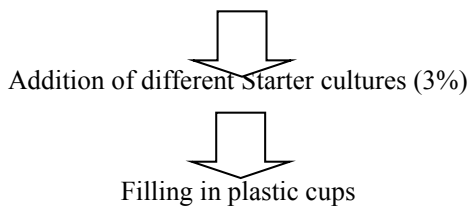

Incubation at suitable temperature until desired $\mathrm{pH}$

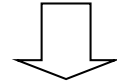

Cooling to room temperature

Shaped into small balls and spread into stainless steel trays

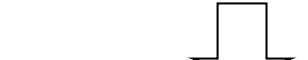

Drying at $60^{\circ} \mathrm{C}$ for $15-18 \mathrm{hrs}$ in an air oven

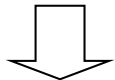

Milled the dried kishk by using a hammer mill

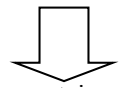

Packed and stored in airtight glass containers at room temperature until used.

Figure 1: Dried kishk-like preparation by using different substrates and starters.

Organic acid determination: The concentrations of organic acids (Lactic, Propionic, acetic, and formic) in different dried Kishk samples were determined by HPLC (Spectra-Physics system, San Jose, CA, USA) method as described by Barrantes et al. [14]. Organic acids were extracted from dried Kishk $(5 \mathrm{~g})$ in a $50 \mathrm{~mL}$ beaker using $25 \mathrm{~mL}$ wateracetonitrile $(1: 4 \mathrm{v} / \mathrm{v})$ (analytical grade, BDH Chemicals Ltd., Poole, UK). The extract after filtration through a Whatman No. 1 filter paper (Whatman Ltd., Maidstone, UK) was injected $(20 \mu \mathrm{L})$ into the HPLC column. The flow rate of the solvent was $0.7 \mu \mathrm{L} \cdot \mathrm{min}^{-1}$ at $65^{\circ} \mathrm{C}$ and the wave length of the detector was $220 \mathrm{~nm}$ [15].

\section{Sensory evaluation}

Organoleptic evaluation was carried out according to AbouDonia et al. [16]. The samples were subjected to organoleptic analysis by 15 well-trained members of the Dairy Science and Technology Department (Fac. Agric. Alexandria Univ., Egypt). The sensory attributes evaluated were: The Flavor (1-45 points), Body and Texture (1-30 points), appearance and Colour (1-15 points) and acidity (1- 10 points). Soups were prepared by adding $20 \mathrm{~g}$ dried kishk to $170 \mathrm{~mL}$ of water and heating with gentle stirring to boiling, simmering for few 
Citation: Nassar KS, Shamsia SM, Attia IA (2016) Improvement of the Nutritional Value of a Cereal Fermented Milk: 2-Dried Kishk Like. J Food Process Technol 7: 638. doi: 10.4172/2157-7110.1000638

Page 3 of 7

min and cooling to $40^{\circ} \mathrm{C}$. The samples $(20 \mathrm{~g})$ were placed in identical glass containers ( $8 \mathrm{~cm}$ diameter, $3.5 \mathrm{~cm}$ height) and served at $40^{\circ} \mathrm{C}$ [17].

\section{Enumeration of microorganisms}

The counts of lactic acid bacteria were enumerated as (CFU/g) using MRS agar according to De man et al. [18]. Proteolytic bacteria, coliforms, yeast and mould were enumerated respectively [19-21]. Whereas aerobic spore forming bacteria were enumerated according to the method described by Harrigan and McCance [22]. Lactobacillus acidophilus was enumerated according to the method described by Lapierra [23].

\section{Statistical analysis}

Statistical analysis was performed by applying three-way ANOVA and multiple comparisons of means of each treatment (cereals, starter cultures and storage time) using the Least Significant Difference (LSD) test at the confidence level of $95 \%$ [24].

\section{Results and Discussion}

\section{Proximate composition}

Proximate analysis of kishk-like products is presented in Tables $2-4$. The results revealed that the effect of cereal type on the proximate analysis of the resultant dried kishk-like products was more pronounced $(P \leq 0.05)$ than that of type of starter culture used.

There were significant differences $(P \leq 0.05)$ in acidity and $\mathrm{pH}$ values between different cereals fermented milk products, depending on the type of cereal or starter culture. The fresh soft cereal fermented

\begin{tabular}{|c|c|c|c|c|c|c|c|c|c|c|}
\hline Samples & Storage period (Days) & $\begin{array}{c}\text { Acidity as lactic } \\
\text { acid }\end{array}$ & $\mathrm{pH}$ & Total Solids $\%$ & Fat content $\%$ & Ash\% & Crude Fiber\% & Crude protein $\%$ & Carbohydrates $\%$ & Salt\% \\
\hline \multirow{5}{*}{ W1 } & Fresh soft pro. & $0.300^{P Q R}$ & $5.44^{\mathrm{A}}$ & $27.42^{\mathrm{RS}}$ & $0.280^{\llcorner}$ & $2.98^{J}$ & $0.36^{\mathrm{E}}$ & $4.99^{\mathrm{K}}$ & $18.80^{\mathrm{NOP}}$ & $2.06^{\mathrm{B}}$ \\
\hline & Fresh dried pro. & $1.803^{\mathrm{IJKL}}$ & $5.23^{\mathrm{D}}$ & $93.27^{\mathrm{A}}$ & $0.95^{\mathrm{A}}$ & $10.15^{\mathrm{A}}$ & $1.21^{\mathrm{B}}$ & $16.97^{\mathrm{ABCDEFG}}$ & $63.98^{\mathrm{CDE}}$ & $7.00^{\mathrm{A}}$ \\
\hline & 30 & $1.880^{\mathrm{HIJK}}$ & $5.17^{\mathrm{EF}}$ & $92.65^{\mathrm{AB}}$ & $0.95^{\mathrm{A}}$ & $10.08^{\mathrm{A}}$ & $1.206^{B}$ & 16.86 $\mathrm{ABCDEFGH}$ & $63.56^{\mathrm{DEF}}$ & $6.96^{\mathrm{A}}$ \\
\hline & 60 & $2.080^{\mathrm{CDEFG}}$ & $5.05^{\lrcorner}$ & $92.25^{\text {BCDEFG }}$ & $0.94^{B}$ & $10.04^{\mathrm{A}}$ & $1.20^{\mathrm{B}}$ & $16.79^{A B C D E F G H}$ & $63.28^{\mathrm{EFG}}$ & $6.93^{\mathrm{A}}$ \\
\hline & 90 & $2.136^{\mathrm{BCDE}}$ & $5.02^{\mathrm{K}}$ & $91.46^{\text {HIJKLM }}$ & $0.933^{\mathrm{BC}}$ & $9.95^{\mathrm{AB}}$ & $1.19^{\mathrm{B}}$ & $16.64^{\mathrm{BCDEFGHI}}$ & $62.74^{\mathrm{FGH}}$ & $6.87^{\mathrm{A}}$ \\
\hline \multirow{5}{*}{ W2 } & Fresh soft pro. & $0.223^{R}$ & $5.23^{\mathrm{D}}$ & $28.92 \mathrm{P}$ & $0.290^{\mathrm{K}}$ & $2.88^{J}$ & $0.36^{\mathrm{E}}$ & $5.03^{K}$ & $20.36^{\mathrm{M}}$ & $2.14^{\mathrm{B}}$ \\
\hline & Fresh dried pro. & $1.963^{\mathrm{FGH}}$ & $5.14^{\mathrm{GH}}$ & $92.35^{\mathrm{BCDE}}$ & $0.94^{\mathrm{B}}$ & $9.206^{\mathrm{GHI}}$ & $1.15^{\mathrm{B}}$ & $16.06^{\mathrm{IJ}}$ & $64.99^{\mathrm{ABC}}$ & $6.85^{\mathrm{A}}$ \\
\hline & 30 & $1.976^{\mathrm{FGH}}$ & $5.12^{\mathrm{H}}$ & $92.29^{\mathrm{BCDEF}}$ & $0.94^{B}$ & $9.20^{\mathrm{GHI}}$ & $1.15^{\mathrm{B}}$ & $16.05^{\mathrm{IJ}}$ & $64.95^{\mathrm{ABC}}$ & $6.85^{\mathrm{A}}$ \\
\hline & 60 & $2.220^{\mathrm{ABC}}$ & $5.01^{\mathrm{K}}$ & $91.86^{\text {CDEFGHIJ }}$ & $0.933^{\mathrm{BC}}$ & $9.16^{\mathrm{GHI}}$ & $1.14^{\mathrm{B}}$ & $15.97^{J}$ & $64.64^{\mathrm{ABCD}}$ & $6.82^{\mathrm{A}}$ \\
\hline & 90 & $2.336 \mathrm{~A}$ & $4.93^{\mathrm{M}}$ & $91.70^{\mathrm{FGHIJKL}}$ & $0.930^{c}$ & $9.14^{\mathrm{GHI}}$ & $1.14^{\mathrm{B}}$ & $15.94^{J}$ & $64.53^{\mathrm{ABCD}}$ & $6.80^{\mathrm{A}}$ \\
\hline \multirow{5}{*}{ W3 } & Fresh soft pro. & $0.226^{\mathrm{QR}}$ & $5.32^{\mathrm{C}}$ & $28.11^{Q}$ & $0.290^{\mathrm{K}}$ & $2.85^{\lrcorner}$ & $0.36^{\mathrm{E}}$ & $5.02^{\mathrm{K}}$ & $19.58^{\mathrm{MN}}$ & $2.10^{B}$ \\
\hline & Fresh dried pro. & $1.896^{\mathrm{HIJ}}$ & $5.19^{\mathrm{E}}$ & $92.28^{\mathrm{BCDEF}}$ & $0.94^{\mathrm{B}}$ & $9.36^{\mathrm{EDFG}}$ & $1.18^{B}$ & $16.48^{\mathrm{FGHIJ}}$ & $64.31^{\mathrm{ABCDE}}$ & $6.91^{\mathrm{A}}$ \\
\hline & 30 & $1.926^{\mathrm{GHI}}$ & $5.16^{\mathrm{FG}}$ & $91.93^{\mathrm{CDEFGHI}}$ & $0.94^{\mathrm{B}}$ & $9.33^{\mathrm{FGH}}$ & $1.17^{\mathrm{B}}$ & $16.42^{\mathrm{GHIJ}}$ & $64.07^{\mathrm{CDE}}$ & $6.88^{\mathrm{A}}$ \\
\hline & 60 & $2.146^{\mathrm{BCD}}$ & $5.08^{\prime}$ & $91.78^{\mathrm{EFGHIJK}}$ & $0.94^{\mathrm{B}}$ & $9.31^{\mathrm{GH}}$ & $1.17^{\mathrm{B}}$ & $16.39^{\mathrm{GHIJ}}$ & $63.96^{\mathrm{CDE}}$ & $6.87^{\mathrm{A}}$ \\
\hline & 90 & $2.226^{A B}$ & $4.92^{\mathrm{M}}$ & $91.33^{\text {IJKLM }}$ & $0.933^{\mathrm{BC}}$ & $9.27^{\mathrm{GHI}}$ & $1.17^{\mathrm{B}}$ & $16.31^{\mathrm{HIJ}}$ & $63.65^{\mathrm{DEF}}$ & $6.84^{\mathrm{A}}$ \\
\hline \multicolumn{2}{|r|}{ SED } & 0.026 & 0.004 & 0.108 & 0.001 & 0.048 & 0.012 & 0.111 & 0.191 & 0.085 \\
\hline \multicolumn{2}{|r|}{ R-Square } & 0.996 & 0.999 & 0.999 & 0.998 & 0.999 & 0.9993 & 0.998 & 0.9997 & 0.996 \\
\hline \multicolumn{2}{|r|}{ Coeff. Var. } & 2.844 & 0.141 & 0.238 & 0.367 & 1.041 & 1.683 & 1.35 & 0.609 & 2.501 \\
\hline
\end{tabular}

Table 2: Chemical properties of cereal fermented milks using burghul from whole wheat.

\begin{tabular}{|c|c|c|c|c|c|c|c|c|c|c|}
\hline Samples & Storage period (Days) & $\begin{array}{l}\text { Acidity as } \\
\text { lactic acid }\end{array}$ & $\mathrm{pH}$ & Total Solids \% & Fat content $\%$ & Ash\% & Crude Fiber\% & Crude protein $\%$ & Carbohydrates $\%$ & Salt $\%$ \\
\hline \multirow{5}{*}{ B1 } & Fresh soft pro. & $0.423^{P}$ & $5.23^{\mathrm{D}}$ & $27.96^{\mathrm{QR}}$ & $0.266^{\mathrm{MN}}$ & $2.86^{J}$ & $0.70^{D}$ & $5.10^{\mathrm{K}}$ & $18.19^{\mathrm{OP}}$ & $2.07^{\mathrm{B}}$ \\
\hline & Fresh dried pro. & $1.910^{\mathrm{HIJ}}$ & $4.97^{L}$ & $91.98^{\mathrm{CDEFGH}}$ & $0.900^{\mathrm{DE}}$ & $9.71^{\mathrm{BC}}$ & $2.38^{\mathrm{A}}$ & $17.31^{\mathrm{A}}$ & $61.68^{\mathrm{HIJK}}$ & $7.01^{\mathrm{A}}$ \\
\hline & 30 & $1.986^{\mathrm{EFGH}}$ & $4.93^{\mathrm{M}}$ & $91.85^{\mathrm{CDEFGHIJ}}$ & $0.900^{\mathrm{DE}}$ & $9.70^{\mathrm{BC}}$ & $2.38^{A}$ & $17.28^{\mathrm{AB}}$ & $61.58^{\mathrm{IJK}}$ & $7.00^{\mathrm{A}}$ \\
\hline & 60 & $2.233^{\mathrm{ABC}}$ & $4.87^{\mathrm{NO}}$ & $91.55^{\text {HIJKLM }}$ & $0.893^{\mathrm{EF}}$ & $9.66^{c}$ & $2.37^{\mathrm{A}}$ & $17.23^{\mathrm{ABC}}$ & $61.39^{\mathrm{IJKL}}$ & $6.98^{\mathrm{A}}$ \\
\hline & 90 & $2.310^{\mathrm{A}}$ & $4.46^{U}$ & $91.43^{\text {HIJKLM }}$ & $0.890^{F}$ & $9.65^{c}$ & $2.37^{A}$ & $17.20^{\mathrm{ABCD}}$ & $61.30^{\mathrm{IJKL}}$ & $6.97^{A}$ \\
\hline \multirow{5}{*}{ B2 } & Fresh soft pro. & $0.440^{P}$ & $4.94^{\mathrm{M}}$ & $26.70^{\top}$ & $0.260^{\mathrm{N}}$ & $2.83^{\jmath}$ & $0.70^{\mathrm{D}}$ & $5.05^{\mathrm{K}}$ & $17.85^{\mathrm{P}}$ & $2.03^{\mathrm{B}}$ \\
\hline & Fresh dried pro. & $1.970^{\mathrm{FGH}}$ & $4.87^{\mathrm{NO}}$ & $91.09^{\mathrm{LMN}}$ & $0.900^{\mathrm{DE}}$ & $9.65^{c}$ & $2.40^{\mathrm{A}}$ & $17.24^{\mathrm{ABC}}$ & $60.90^{\mathrm{JKL}}$ & $6.95^{\mathrm{A}}$ \\
\hline & 30 & $2.086^{\mathrm{CDEF}}$ & $4.74^{\mathrm{Q}}$ & $90.96^{\mathrm{MN}}$ & $0.900^{\mathrm{DE}}$ & $9.64^{c}$ & $2.39^{\mathrm{A}}$ & $17.21^{\mathrm{ABCD}}$ & $60.81^{\mathrm{JKL}}$ & $6.93^{\mathrm{A}}$ \\
\hline & 60 & $2.250^{\mathrm{AB}}$ & $4.66^{\mathrm{s}}$ & $90.63^{\text {NO }}$ & $0.890^{\mathrm{F}}$ & $9.62^{\mathrm{CDE}}$ & $2.38^{\mathrm{A}}$ & $17.15^{\mathrm{ABCDE}}$ & $60.58^{\mathrm{KL}}$ & $6.91^{\mathrm{A}}$ \\
\hline & 90 & $2.336^{A}$ & $4.52^{\top}$ & $90.30^{\circ}$ & $0.890^{\mathrm{F}}$ & $9.59^{\mathrm{CDEF}}$ & $2.37^{\mathrm{A}}$ & $17.09^{\mathrm{ABCDEF}}$ & $60.35^{\llcorner}$ & $6.88^{A}$ \\
\hline \multirow{5}{*}{ B3 } & Fresh soft pro. & $0.413^{\mathrm{PQ}}$ & $5.43^{\mathrm{AB}}$ & $27.29^{\mathrm{ST}}$ & $0.270^{\mathrm{M}}$ & $2.85^{\mathrm{J}}$ & $0.70^{\mathrm{D}}$ & $5.10^{\mathrm{K}}$ & $17.94^{\mathrm{P}}$ & $2.03^{\mathrm{B}}$ \\
\hline & Fresh dried pro. & $1.926^{\mathrm{GHI}}$ & $5.22^{\mathrm{D}}$ & $92.69^{\mathrm{AB}}$ & $0.903^{\mathrm{D}}$ & $9.70^{\mathrm{BC}}$ & $2.38^{A}$ & $17.32^{\mathrm{A}}$ & $62.38^{\mathrm{GH}}$ & $6.88^{A}$ \\
\hline & 30 & $2.033^{\mathrm{DEFGH}}$ & $5.07^{1 J}$ & $92.21^{\text {BCDEFG }}$ & $0.900^{\mathrm{DE}}$ & $9.65^{c}$ & $2.37^{\mathrm{A}}$ & $17.23^{\mathrm{ABC}}$ & $62.05^{\mathrm{HI}}$ & $6.85^{\mathrm{A}}$ \\
\hline & 60 & $2.190^{\mathrm{ABC}}$ & $5.01^{\mathrm{K}}$ & 91.84 DEFGHIJK & $0.896^{\mathrm{DEF}}$ & $9.61^{\mathrm{CDE}}$ & $2.36^{\mathrm{A}}$ & $17.16^{\mathrm{ABCDE}}$ & $61.80^{\mathrm{HIJ}}$ & $6.82^{\mathrm{A}}$ \\
\hline & 90 & $2.266^{A B}$ & $4.89^{\mathrm{N}}$ & $91.64^{\mathrm{GHIJKL}}$ & $0.890^{F}$ & $9.62^{\mathrm{CD}}$ & $2.35^{\mathrm{A}}$ & $17.12^{\mathrm{ABCDE}}$ & $61.64^{\mathrm{HIJK}}$ & $6.81^{\mathrm{A}}$ \\
\hline \multicolumn{2}{|r|}{ SED } & 0.026 & 0.004 & 0.108 & 0.001 & 0.048 & 0.012 & 0.111 & 0.191 & 0.085 \\
\hline \multicolumn{2}{|r|}{ R-Square } & 0.996 & 0.999 & 0.999 & 0.998 & 0.999 & 0.9993 & 0.998 & 0.9997 & 0.996 \\
\hline \multicolumn{2}{|r|}{ Coeff. Var. } & 2.844 & 0.141 & 0.238 & 0.367 & 1.041 & 1.683 & 1.35 & 0.609 & 2.501 \\
\hline
\end{tabular}

Table 3: Chemical properties of cereal fermented milks using burghul from whole barely. 
Citation: Nassar KS, Shamsia SM, Attia IA (2016) Improvement of the Nutritional Value of a Cereal Fermented Milk: 2-Dried Kishk Like. J Food Process Technol 7: 638. doi: 10.4172/2157-7110.1000638

Page 4 of 7

\begin{tabular}{|c|c|c|c|c|c|c|c|c|c|c|}
\hline Samples & Storage period (Days) & $\begin{array}{l}\text { Acidity as lactic } \\
\text { acid }\end{array}$ & $\mathrm{pH}$ & Total Solids \% & Fat content $\%$ & Ash\% & Crude Fiber\% & Crude protein $\%$ & Carbohydrates $\%$ & Salt $\%$ \\
\hline \multirow{5}{*}{ F1 } & Fresh soft pro. & $0.306^{P Q R}$ & $5.41^{\mathrm{B}}$ & $27.20^{S T}$ & $0.220^{\mathrm{P}}$ & $2.72^{\mathrm{J}}$ & $0.27^{\mathrm{F}}$ & $4.93^{\mathrm{K}}$ & $19.05^{\mathrm{NO}}$ & $2.03^{B}$ \\
\hline & Fresh dried pro. & $1.490^{\circ}$ & $5.24^{\mathrm{D}}$ & $92.46^{\mathrm{BCD}}$ & $0.733^{\prime}$ & $9.24^{\mathrm{GHI}}$ & $0.93^{c}$ & $16.77^{\mathrm{ABCDEFGH}}$ & $64.77^{\mathrm{ABC}}$ & $6.90^{\mathrm{A}}$ \\
\hline & 30 & $1.596^{\mathrm{NO}}$ & $5.09^{1}$ & $91.67^{F G H I J K L}$ & 0.7301 & $9.166^{\mathrm{GHI}}$ & $0.92^{\mathrm{C}}$ & $16.63^{\mathrm{CDEFGHI}}$ & $64.22^{\mathrm{BCDE}}$ & $6.85^{\mathrm{A}}$ \\
\hline & 60 & $1.690^{\mathrm{LMN}}$ & $5.01^{\mathrm{K}}$ & $91.36^{\text {HIJKLM }}$ & $0.730^{\prime}$ & $9.136^{\mathrm{GHI}}$ & $0.92^{\mathrm{C}}$ & $16.58^{\text {DEFGHIJ }}$ & $64.00^{\mathrm{CDE}}$ & $6.82^{\mathrm{A}}$ \\
\hline & 90 & $1.790^{\mathrm{IJKL}}$ & $4.92^{\mathrm{M}}$ & $91.22^{\text {KLMN }}$ & $0.720 \mathrm{~J}$ & $9.12^{\mathrm{GHI}}$ & $0.92^{c}$ & $16.55^{\text {EFGHIJ }}$ & $63.90^{\mathrm{CDE}}$ & $6.80^{\mathrm{A}}$ \\
\hline \multirow{5}{*}{ F2 } & Fresh soft pro. & $0.296^{\mathrm{PQR}}$ & $5.33^{\mathrm{C}}$ & $27.93^{\mathrm{QR}}$ & $0.230^{\circ}$ & $2.74^{\mathrm{J}}$ & $0.27^{F}$ & $4.94^{\mathrm{K}}$ & $19.74^{\mathrm{MN}}$ & $2.05^{\mathrm{B}}$ \\
\hline & Fresh dried pro. & $1.593^{\mathrm{NO}}$ & $5.14^{\mathrm{GH}}$ & $92.47^{\mathrm{BC}}$ & $0.750^{\mathrm{H}}$ & $9.09^{\mathrm{GHI}}$ & $0.91^{\mathrm{C}}$ & $16.37^{\text {GHIJ }}$ & $65.33^{A}$ & $6.81^{\mathrm{A}}$ \\
\hline & 30 & $1.740^{\text {KLMN }}$ & $5.08^{1}$ & $92.28^{\mathrm{BCDEF}}$ & $0.750^{\mathrm{H}}$ & $9.07^{\mathrm{HI}}$ & $0.91^{\mathrm{C}}$ & $16.34^{\mathrm{GHIJ}}$ & $65.20^{\mathrm{AB}}$ & $6.79^{A}$ \\
\hline & 60 & $1.806^{\mathrm{IJKL}}$ & $4.82^{\mathrm{P}}$ & $91.89^{\mathrm{CDEFGHI}}$ & $0.750^{\mathrm{H}}$ & $9.13^{\mathrm{GHI}}$ & $0.90^{\mathrm{C}}$ & $16.27^{\mathrm{HIJ}}$ & $64.82^{\mathrm{ABC}}$ & $6.76^{\mathrm{A}}$ \\
\hline & 90 & $1.940^{\mathrm{FGHI}}$ & $4.70^{R}$ & $91.72^{\text {EFGHIJK }}$ & $0.750^{\mathrm{H}}$ & $9.02^{1}$ & $0.90^{c}$ & $16.24^{\mathrm{HIJ}}$ & $64.80^{A B C}$ & $6.66^{\mathrm{A}}$ \\
\hline \multirow{5}{*}{ F3 } & Fresh soft pro. & $0.310^{P Q R}$ & $5.41^{\mathrm{B}}$ & $27.12^{\text {ST }}$ & $0.290^{\circ}$ & $2.86^{\lrcorner}$ & $0.27^{F}$ & $4.92^{\mathrm{K}}$ & $19.67^{\mathrm{MN}}$ & $2.05^{\mathrm{B}}$ \\
\hline & Fresh dried pro. & $1.500^{\circ}$ & $5.05^{\mathrm{J}}$ & 91.44 HIJKLM & $0.766^{\mathrm{G}}$ & $9.35^{\mathrm{EDFGH}}$ & $0.89^{\mathrm{c}}$ & $16.11^{1 \mathrm{~J}}$ & $64.31^{\mathrm{ABCDE}}$ & $6.71^{\mathrm{A}}$ \\
\hline & 30 & $1.516^{\circ}$ & $5.02^{\mathrm{K}}$ & $91.34^{\mathrm{IJKLM}}$ & $0.763^{G}$ & $9.34^{\text {EDFGH }}$ & $0.88^{\mathrm{C}}$ & $16.09^{\mathrm{IJ}}$ & $64.25^{\mathrm{ABCDE}}$ & $6.71^{\mathrm{A}}$ \\
\hline & 60 & $1.623^{\mathrm{MNO}}$ & $4.88^{\mathrm{N}}$ & $91.32^{\text {IJKLM }}$ & $0.760^{G}$ & $9.34^{\text {EDFGH }}$ & $0.88^{\mathrm{C}}$ & $16.09^{I J}$ & $64.24^{\mathrm{ABCDE}}$ & $6.70^{\mathrm{A}}$ \\
\hline & 90 & $1.766^{\mathrm{JLLM}}$ & $4.85^{\circ}$ & $91.25^{\text {JKLMN }}$ & $0.760^{\mathrm{G}}$ & $9.333^{\mathrm{FGH}}$ & $0.88^{\mathrm{C}}$ & $16.08^{I J}$ & $64.18^{\mathrm{BCDE}}$ & $6.67^{\mathrm{A}}$ \\
\hline \multicolumn{2}{|r|}{ SED } & 0.026 & 0.004 & 0.108 & 0.001 & 0.048 & 0.012 & 0.111 & 0.191 & 0.085 \\
\hline \multicolumn{2}{|r|}{ R-Square } & 0.996 & 0.999 & 0.999 & 0.998 & 0.999 & 0.9993 & 0.998 & 0.9997 & 0.996 \\
\hline \multicolumn{2}{|r|}{ Coeff. Var. } & 2.844 & 0.141 & 0.238 & 0.367 & 1.041 & 1.683 & 1.35 & 0.609 & 2.501 \\
\hline
\end{tabular}

Table 4: Chemical properties of cereal fermented milks using burghul from freek (green wheat).

milk products containing Barely (B2, B1 and B3, Respectively) were characterized by higher acidity as compared with their containing of freek (F3, F1 and F2) and wheat (W1, W3 and W2, Respectively). These results are in agreement with [9,16,25-28]. There is no significant difference $(P \leq 0.05)$ between the values of ash, salt, protein and carbohydrate of kishk-like before draying. While, ranged as follow: (2.72-2.98), (2.03-2.14), (4.92-5.10) and (17.85-20.36), respectively. The crude fibers content was significant $(P \leq 0.05)$ depending on the type of cereal used. Whereas, the barley kishk products had higher its values $(0.70 \%)$ than other samples, this is due to the higher content of crude fiber which reached $2.51 \%[9,25]$. As expected the type of starter culture used in the fermentation did not effect on the total solids and fat contents but the variation could be attributed to the fat content of kishk components and blends. These results were in agreement with those obtained by $[2,9,25,29]$.

There was a significant $(\mathrm{P} \leq 0.05)$ increase in $\mathrm{pH}$ and acidity percentages for all fresh samples incorporation with the corresponding values of dry products (Tables 2-4). However, the samples of dried cereal fermented milk products which fermented with Yoghurt and Bio-Yoghurt starter (2) were characterized with higher acidity rates than either fermented with mixed cultures contained only Yoghurt starter culture (1) or fermented by mixed cultures of Yoghurt starter culture and Lactobacillus plantarum (3), respectively. During storage at $\left(25 \pm 2^{\circ} \mathrm{C}\right)$ for 90 days, significant decrease $(P \leq 0.05)$ were recorded in $\mathrm{pH}$ of different dried kishk-like products. Moreover, gradual decrease in $\mathrm{pH}$ could be observed in all samples, with extending the storage period ( 3 months), that due to limit growth of various bacterial starter cultures and the slow fermentation of residual lactose [9,25,29-31].

Dried kishk-like samples had slightly decrease in total solids, fat, Ash, crude fibers, protein, carbohydrate and salt contents until the end of storage period [16,31]. The ranges of previous contents were as follow: (90.30\% to $93.27 \%),(0.720 \%$ to $0.950 \%),(9.02 \%$ to $10.15 \%)$, ( $0.89 \%$ to $2.40 \%),(15.94 \%$ to $17.31 \%),(60.35 \%$ to $65.33 \%)$ and $(6.66 \%$ to $7 \%)$ respectively. These results are in agreement with previous studies [2,16,31-35].

\section{Colour of kishk samples}

Data presented in Table 3 show the colour of dried kishk samples as obtained using Lovibond Tintometer. The values of the primary Colours (Red, yellow and blue) showed that all dried kishk samples as ranged (3 - 5), (6 - 6.9) and (1.9 - 4.9), respectively. The data for primary colours reflects the values of $\mathrm{X}, \mathrm{Y}$ and $\mathrm{Z}$ coordinates. The $\mathrm{X}$ value for (B1) treatment had significantly higher than among of samples (0.395) while the (F3) sample had the highest value of Y coordinate (0.544). Whereas, the $\mathrm{Z}$ coordinate showed the opposite figure. As a result of saturation and visual density, the brightness of (W1) sample was the highest percentage $(66.07 \%)$. on contrary, (F3) treatment had lower brightness $(42.66 \%)$ than other samples. These results are in agreements with Toufeli et al. [17], Bilgicli and Ibanoglu [36].

\section{Organic acids determination}

The concentrations ( $p p m)$ of organic acids (Lactic acid, Propionic acid, Acetic acid and formic acid) in the dried Kishk samples after 90 days of storage made with wheat, barley and Freek Burghul Skim milk is shown in Table 4. Lactic, propionic and acetic acid formed during lactic fermentation. The (W1) treatment had the highest levels of Propionic, Acetic and Lactic acids respectively, except formic acid comparing to among of samples [6,15]. Furthermore, propionic acid had the lowest or not detected in kishk samples made with barley, and these results are in agreement with Tamime et al. [37].

\section{Microbiological analysis}

Microbiological composition of kishk-like products are shown in Table 5. To produce healthy and safety of kishk that it's based on the critical control points during the making of kishk were cooking, fermentation, drying and storage $[35,38]$. The fermented fresh soft products had 2.30-2.48 and 1.60-2.44 c.f.u $\times 10^{-3} / \mathrm{gm}$. for lactic acid bacteria and Lactobacillus acidophilus, respectively. However, the drying treatment gets rid of the bacterial starter, and these results agreement with $[2,16]$. Furthermore, all the samples did not contain any growth in $0.1 \mathrm{gm}$ on SDA, VRBA, NA and MSA media in either fresh or dried products through the storage period. These results are revealed 
Citation: Nassar KS, Shamsia SM, Attia IA (2016) Improvement of the Nutritional Value of a Cereal Fermented Milk: 2-Dried Kishk Like. J Food Process Technol 7: 638. doi: 10.4172/2157-7110.1000638

Page 5 of 7

\begin{tabular}{|c|c|c|c|c|c|c|c|c|c|}
\hline Samples & Red & Yellow & Blue & $\mathbf{X}$ & $\mathbf{Y}$ & $\mathbf{Z}$ & Visual density & Saturation\% & Brightness\% \\
\hline W1 & 3 & 6 & 1.9 & 0.355 & 0.415 & 0.23 & 0.18 & 38.64 & 66.07 \\
\hline W2 & 4 & 6 & 2.6 & 0.34 & 0.425 & 0.235 & 0.23 & 45.45 & 58.88 \\
\hline W3 & 4 & 6 & 2.7 & 0.355 & 0.475 & 0.19 & 0.235 & 59.57 & 58.21 \\
\hline B1 & 4 & 6 & 2.7 & 0.395 & 0.415 & 0.19 & 0.235 & 59.57 & 58.21 \\
\hline B2 & 4.6 & 6.9 & 3.3 & 0.385 & 0.465 & 0.15 & 0.28 & 66.67 & 52.48 \\
\hline B3 & 4.4 & 6.9 & 3.3 & 0.35 & 0.53 & 0.12 & 0.25 & 70.21 & 56.23 \\
\hline F1 & 5 & 6.9 & 3.9 & 0.36 & 0.53 & 0.11 & 0.33 & 79.07 & 46.77 \\
\hline F2 & 4.6 & 6.9 & 3.9 & 0.36 & 0.525 & 0.115 & 0.35 & 70.21 & 44.76 \\
\hline F3 & 4.9 & 6.9 & 4.9 & 0.356 & 0.544 & 0.1 & 0.37 & 83.72 & 42.66 \\
\hline
\end{tabular}

B: Dried kishk like manufactured from fermented whole barley burghul skim milk.

F: Dried kishk like manufactured from fermented freek burghul skim milk.

W: Dried kishk-like manufactured from fermented whole wheat burghul skim milk.

Starters: 1: (3\% Yoghurt starter); 2: (2\% Yoghurt starter + 1\% Bio-yoghurt starter); 3: (2\% Yoghurt starter + 1\% Lactobacillus plantarum).

Table 5: Colour of dried kishk-like samples as measured by Lovibond and C.I.E system.

\begin{tabular}{|c|c|c|c|c|}
\hline \multirow[b]{2}{*}{ Samples } & \multicolumn{4}{|c|}{ Organic acids } \\
\hline & $\begin{array}{c}\text { Lactic acid } \\
\text { ppm }\end{array}$ & $\begin{array}{l}\text { Propionic acid } \\
\quad p p m\end{array}$ & Acetic acid $p p m$ & Formic acid $p p m$ \\
\hline W1 & 1134.57 & 3522.16 & 2930.35 & $<50$ \\
\hline W2 & 908.94 & 3341.66 & 2728.21 & $<50$ \\
\hline W3 & 901.86 & 3451.5 & 973.285 & $<50$ \\
\hline B1 & 903.9 & $<50$ & 2147.14 & $<50$ \\
\hline B2 & 889.16 & $<50$ & $<50$ & 320.05 \\
\hline B3 & 717.5 & $<50$ & 2153.64 & 366.248 \\
\hline $\mathrm{F} 1$ & 601.25 & 562.33 & $<50$ & 738.954 \\
\hline $\mathrm{F} 2$ & 691.52 & 562.7 & 481.357 & $<50$ \\
\hline F3 & 638.33 & 598.72 & $<50$ & $<50$ \\
\hline \multicolumn{5}{|c|}{$\begin{array}{l}\text { B: Dried kishk-like manufactured from fermented whole barley burghul skim milk. } \\
\text { F: Dried kishk-like manufactured from fermented freek burghul skim milk. } \\
\text { W: Dried kishk-like manufactured from fermented whole wheat burghul skim milk. } \\
\text { Starter: 1: ( } 3 \% \text { Yoghurt starter), 2: ( } 2 \% \text { Yoghurt starter }+1 \% \text { Bio-yoghurt starter) and 3: ( } 2 \% \text { Yoghurt starter }+1 \% \text { Lactobacillus plantarum). }\end{array}$} \\
\hline
\end{tabular}

Table 6: Organic acids concentrations of dried cereal fermented milks.

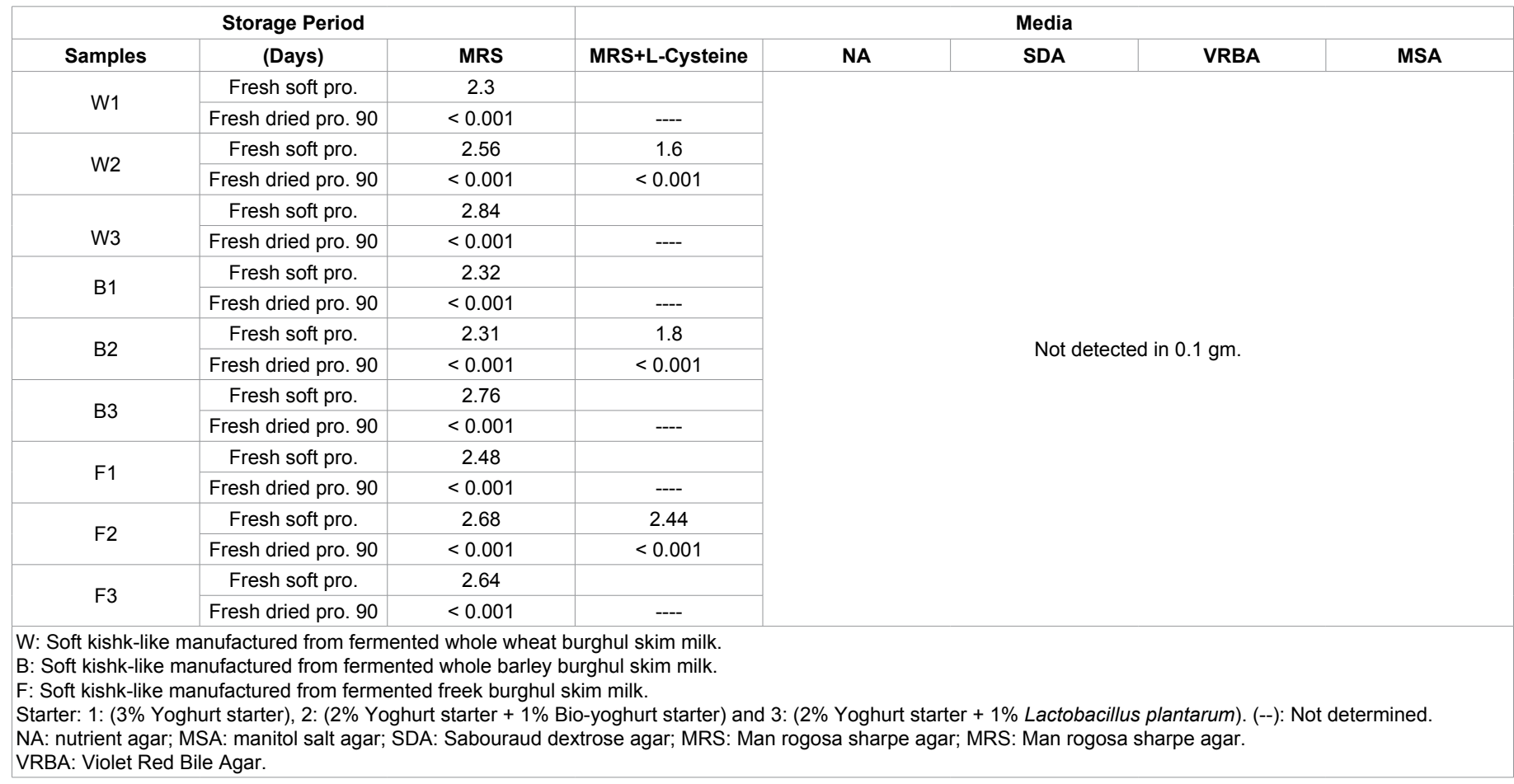

Table 7: Changes in viable microbial counts (c.f.u $\times 10^{-3} / \mathrm{gm}$.) in dried cereal fermented milks. 
Citation: Nassar KS, Shamsia SM, Attia IA (2016) Improvement of the Nutritional Value of a Cereal Fermented Milk: 2-Dried Kishk Like. J Food Process Technol 7: 638. doi: 10.4172/2157-7110.1000638

the good hygiene sanitation during manufacture different products. The low $\mathrm{pH}$ (4.46-5.23), release of organic acids due to fermentation, salt additive and low moisture content (6.73\% to $9.70 \%)$ lead to a harsh environment (bacteriostatic effect) for pathogenic microorganisms, in which food spoilage may not occur and shelf life increases [35].

\section{Sensory evaluation of recombined dried kishk like}

Sensorial evaluation of recombined dried kishk samples is given in Tables 6-8. There were no significant differences $(P \leq 0.05)$ between the soup samples in flavour and body and texture scores. However, significant difference existed with regard to appearance and color and acidity, with barley skimmed milk Kishk soup having the lowest score. It was clear that, the addition of wheat or freek burghul didn't affect the

\begin{tabular}{|c|c|c|c|c|c|}
\hline Sample & Flavour -45 & $\begin{array}{c}\text { Body I } \\
\text { texture -30 }\end{array}$ & $\begin{array}{c}\text { Appearance } \\
\text { and colour } \\
-15\end{array}$ & Acidity -10 & Total -100 \\
\hline W1 & $38^{\mathrm{AB}}$ & $25^{\mathrm{A}}$ & $13^{\mathrm{A}}$ & $7^{\mathrm{BC}}$ & $83^{\mathrm{A}}$ \\
\hline $\mathrm{W} 2$ & $39^{\mathrm{AB}}$ & $25^{\mathrm{A}}$ & $12^{\mathrm{A}}$ & $7^{\mathrm{BC}}$ & $83^{\mathrm{A}}$ \\
\hline $\mathrm{W} 3$ & $40^{\mathrm{A}}$ & $26^{\mathrm{A}}$ & $11^{\mathrm{A}}$ & $6^{\mathrm{C}}$ & $82^{\mathrm{A}}$ \\
\hline $\mathrm{B} 1$ & $33^{\mathrm{C}}$ & $24^{\mathrm{A}}$ & $8^{\mathrm{B}}$ & $6^{\mathrm{C}}$ & $71^{\mathrm{B}}$ \\
\hline $\mathrm{B} 2$ & $36^{\mathrm{BC}}$ & $24^{\mathrm{A}}$ & $8^{\mathrm{B}}$ & $5^{\mathrm{D}}$ & $70^{\mathrm{B}}$ \\
\hline $\mathrm{B} 3$ & $34^{\mathrm{C}}$ & $24^{\mathrm{A}}$ & $9^{\mathrm{B}}$ & $6^{\mathrm{C}}$ & $74^{\mathrm{B}}$ \\
\hline F1 & $40^{\mathrm{A}}$ & $25^{\mathrm{A}}$ & $13^{\mathrm{A}}$ & $9^{\mathrm{A}}$ & $87^{\mathrm{A}}$ \\
\hline F2 & $40^{\mathrm{A}}$ & $24^{\mathrm{A}}$ & $12^{\mathrm{A}}$ & $8^{\mathrm{AB}}$ & $84^{\mathrm{A}}$ \\
\hline F3 & $40^{\mathrm{A}}$ & $24^{\mathrm{A}}$ & $13^{\mathrm{A}}$ & $8^{\mathrm{AB}}$ & $85^{\mathrm{A}}$ \\
\hline SED & 0.555 & 0.368 & 0.43 & 0.248 & 0.881 \\
\hline R-Square & 0.897 & 0.53 & 0.928 & 0.883 & 0.95 \\
\hline Coeff. Var. & 2.534 & 2.583 & 6.663 & 6.246 & 1.891 \\
\hline W D & & 2.53 & & & \\
\hline
\end{tabular}

W: Dried kishk-like manufactured from fermented whole wheat burghul skim milk. B: Dried kishk-like manufactured from fermented whole barley burghul skim milk. F: Dried kishk-like manufactured from fermented freek burghul skim milk.

Starter: 1: (3\% Yoghurt starter); 2 : (2\% Yoghurt starter + 1\% Bio-yoghurt starter); 3: $(2 \%$ Yoghurt starter $+1 \%$ Lactobacillus plantarum $)$

SED: Standard Error of Difference.

Table 8: Organoleptic properties of recombined mixtures.

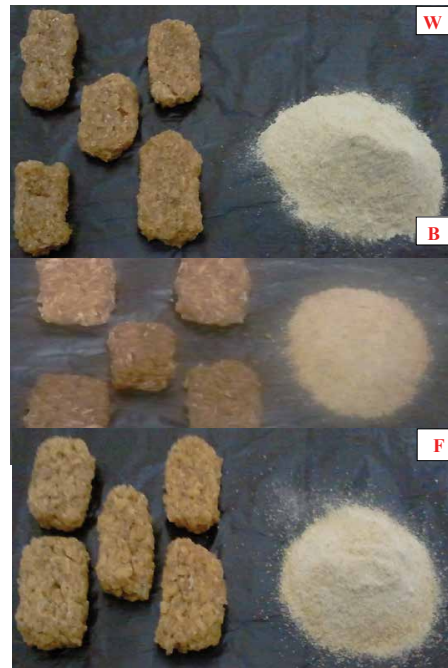

Figure 2: The pictures of dried Kishk-like products.

W: Dried kishk-like manufactured from fermented whole wheat burghul Skim milk with different types of starter cultures.

B: Dried kishk-like manufactured from fermented whole barley burghul Skim milk with different types of starter cultures.

F: Dried kishk-like manufactured from fermented freek burghul Skim milk with different types of starter cultures. general acceptability of them, whereas the addition of barley burghul had lowering the total score acceptability (Figure 2) [16,17,37]. These products could be used to feed infants to 6 months as a complementary diet, children and elderly persons who need special care instead of the commercial extracts because the nutritive value of cereal fermented milks higher than of cereal alone. On the other hand the therapeutic effect of crude fibers and wheat bran in diets [16] (Tables 7 and 8).

\section{Conclusion}

The cereal fermented skim milk shown long shelf-life without changing in either chemical or microbial characteristics during the storage period at room temperature. This phenomenon is accepted as result of those mixtures.

\section{References}

1. Blandino A, Al-Aseeri ME, Pandiella SS, Cantero D, Webb C (2003) Cerealbased fermented foods and beverages. Food Res Int 36: 527-543.

2. Attia IA, Khattab AA (1985) Microbiological and chemical studies on Kishk. J Alex Sci Exch 6: 63-71.

3. Abou-Zeid NA (2016) Review of Egyptian cereal-based fermented product (Kishk). Int J Agri Innov Res 4: 600-609.

4. Tamime AY, Muir DD, Khaskheli M, Barclay MNI (2000) Effect of processing conditions and raw materials on the properties of Kishk: 1. Compositional and microbiological qualities. Ebensmittel-Wissenschaft Technol 33: 444-451.

5. El-Gindy SM (1983) Fermented foods of Egypt and Middle East. J Food Protection 46: 358-567.

6. Tamime AY, O'connor TP (1995) Kishk-A dried fermented milk/cereal mixture. Int Dairy J 5: 109-128.

7. Tamime AY, Muir DD, Barclay MNI, Khaskheli M, Mcnulty D (1997) Laboratorymade Kishk from wheat, oat and barley: 1. Production and comparison of chemical and nutritional composition of Burghol. Food Res Int 30: 311-317.

8. Kurmann JA, Rasic JL, Kroger M (1992) Encyclopedia of fermented fresh milk products. Van Nostrand Reinhold, New York.

9. Nassar KS, Shamsia SM, Attia IA (2016) Improvement of the nutritional value of cereal fermented milk: 1- Soft Kishk like. J Food Process Technol 7: 1.

10. AOAC (2007) Official Methods of Analysis (18thedn). Association of Official Analytical Chemists, Washington DC, USA.

11. Ling ER (1963) Text book of Dairy chemistry. Chapman and Hall.

12. FAO (2003) Food energy-methods of analysis and conversion factors. FAO Food and Nutrition Paper 77. Food and Agriculture Organization of the United Nations, Rome.

13. AOCS (1990) American Oil Chemist's Society. Official methods and recommended practice of the American oil chemist's society.

14. Barrantes E, Tamime AY, Sword AM, Muir DD, Kalab M (1996) The manufacturer of set type natural yogurt containing different oils: 1 . Compositional quality, microbiological evaluation and organoleptic properties. Int Dairy J 6: 811-826.

15. Tamime AY, Barclay MNI, Amarowicz R, Mcnulty D (1999) Kishk-a dried fermented milk /cereal mixture: 1. Composition of gross components, carbohydrates, organic acids and fatty acids. Lait 79: 317-330.

16. Abou-Donia SA, Attia IA, Khattab AA, EL-Shenawi Z (1991) Formulation of dried cereal fermented milks with prolonged storage life. Egyptian J Dairy Sci 19: 283-299.

17. Toufeili I, Melki C, Shadarevian S, Robinson RK (1999) Some nutritional and sensory properties of bulgur and whole wheat meal kishk (a fermented milkwheat mixture). J Food Qual Pref 10: 9-15.

18. De Man JC, Rogosa M, Sharp EM (1960) A medium for the cultivation of lactobacilli. J Appl Bacteriol 22: 130-134.

19. APHA (1992) American Public Health Association, standard method for the examination of dairy products (16thedn). Washington DC, USA.

20. IDF (1985) Milk and milk products-enumeration of coliform, colony count technique and most probable number technique at $30^{\circ} \mathrm{C}$, standard $73 \mathrm{~A}$ International Dairy Federation, Brussels. 
Citation: Nassar KS, Shamsia SM, Attia IA (2016) Improvement of the Nutritional Value of a Cereal Fermented Milk: 2-Dried Kishk Like. J Food Process Technol 7: 638. doi: 10.4172/2157-7110.1000638

Page 7 of 7

21. IDF (1990) Milk and milk products-Enumeration of yeasts and moulds colony count at $25^{\circ} \mathrm{C}$, standard 94B. International Dairy Federation, Brussels.

22. Harrigan WF, McCance ME (1960) Laboratory methods in food and microbiology (Revised edition). Academic Press, London.

23. Lapierra LP, Undeland P, Cox LJ (1992) Lithium chloride-sodium propionate agar for the enumeration of Bifidobacteria in fermented dairy products. J Dairy Sci 75: 1192.

24. SAS (2013) Statistical analysis system user guide, Version 9.3. SAS Institute Inc, Cary, NC, USA.

25. Hussein GAM (2011) Production and properties of some cereal-based functional fermented dairy products. Egyptian J Dairy Sci 39: 89-100.

26. Mehanna AS, Hefnawy SA (1990) A study to follow the chemical changes during processing and storage of zabadi. Egypt J Dairy Sci 18: 425-434.

27. Mehanna AS (1991) An attempt to improve some properties of zabadi by applying low temperature long incubation period in the manufacturing process. Egypt Dairy Sci 19: 221-229.

28. Kailasapathy K, Rybka S (1997) Lactobacillus acidophilus and Bifidobacterium spp. Their therapeutic potential and survival in yoghurt. Australian J Dairy Tech 52: 28.

29. Barrantes E, Tammime AY, Muir DD, Swoed AM (1994) The effect of substitution of fat by microparticulated whey protein on the quality of set-type natural yoghurt. J Soci Dairy Technol 47: 61.

30. Aklain AS (1996) L (+), D (-) Lactic acid content and aroma profile in bioghurt, bifigurt, biograde in comparison with yogurt. Egypt J Dairy Sci 24: 227.
31. El-Nawawy MA, Ibrahim R, AI-Bonayan AM, El-Beialy AR (2012) Development of functional food products. Int J Diary Sci Res 1: 12-17

32. Tamime YA, Barclay MNI, Amarowicz R, McNulty D (1999) Kishk-a dried fermented milk/cereal mixture.1. Composition of gross components, carbohydrates, organic acids and fatty acids. Lait 79: 317-330.

33. Tamime AY, Barclay MNI, Law AJR, Leaver G, Anifantakis EM, et al. (1999) Kishk-a dried fermented milk/cereal mixture: 2. Assessment of a variety of protein analytical techniques for determining adulteration and proteolysis. Lait 79: 331-339.

34. Tamime YA, Muir DD, Kaskheli M, Barclay MNI (2000) Effect of processing conditions and raw materials on the properties of Kishk: 1 . Compositional and microbiological qualities. Lebensm Wiss Technol 33: 444-451.

35. Mashak Z, Hamidreza S, Banafsheh M, Shahram N (2014) Chemical and microbial properties of two Iranian traditional fermented cereal-dairy based foods: Kashk-e-Zard and Tarkhineh. Int J Biosci 4: 124-133.

36. Bilgicli N, Ibanoglu S (2007) Effect of wheat germ and wheat bran on the fermentation activity, Phytic acid content and colour of tarhana, a wheat flouryoghurt mixture. J Food Eng 78: 681-686.

37. Tamime AY, Muir DD, Barclay MNI, Khaskheli M, McNulty D (1997) Laboratorymade Kishk from wheat, oat and barley: 2. Compositional quality and sensory properties. Food Res Int 30: 319-326.

38. Dalgıc AC, Belibagli KB (2008) Hazard analysis critical control points implementation in traditional foods: a case study of Tarhana processing. Int $J$ Food Sci Technol 43: 1352-1360. 